\title{
Micosis por Pythium Insidiosum primer caso con diagnóstico definitivo en Colombia
}

\author{
Edward Javier Acero-Mondragón MDV. Esp. MSc. ${ }^{1,2}$, Daniel Alfonso \\ Botero Rosas, MD. PhD ${ }^{1}$, María Inés Maldonado Arango MD MSc ${ }^{1}$.
}

\author{
${ }^{1}$ Universidad de La Sabana, Facultad de Medicina, Área de Morfofisiología, \\ Grupo Proseim, Chía-Cundinamarca, Colombia. \\ ${ }^{2}$ Universidad Militar "Nueva Granada” Facultad de Medicina, Área de Morfología, \\ Grupo Morfología Médica, Bogotá, Colombia.
}

Correspondencia: edward.acero@unisabana.edu.co, edward.acero@unimilitar.edu.co

Recibido: 14/06/2013 Aceptado:27/06/2013

\begin{abstract}
Resumen
Pythium insidiosum es un hongo distribuido globalmente causante de una micosis emergente llamada Pitiosis en personas y animales. El diagnóstico definitivo involucra correlación clínico epidemiológica, clínico patológica, histopatológica y molecular. En Colombia no existen antecedentes diagnósticos de Pitiosis con estas correlaciones. Pitiosis fue demostrada dos equinos de zona rural en Puerto Boyacá, Departamento de Boyacá-Colombia. Los equinos presentaron en extremidades lesiones únicas, pruriginosas, ulceradas, granulomatosas, alternada de masas sólidas con secreción serosanguinolenta. De las biopsias los hallazgos anatomopatológicos demostraron masas sólidas de aspecto coralino, las cuales se procesaron histopatológicamente. Los resultados correlacionados de biogeografía, las lesiones halladas en los equinos y en ellas los patrones histopatológicos de Splendore-Hoeplii, con hallazgos de hifas raramente septadas con diámetro de 2,5 a 6,2 micras en ramificación angulada, junto a la secuenciación del DNA extraído y comparado con el depositado para P.insidiosum en el GenBank permitió demostrar en todas las muestras una homología del 100\% conformándose por primera vez el diagnóstico definitivo de Pitiosis en Colombia.

Palabras Clave: Pythyum insidiosum, Pitiosis, diagnóstico.
\end{abstract}

\section{Mycoses for Pythium insidiosum. First case with definitive diagnosis in Colombia}

\begin{abstract}
Pythium insidiosum is a globally distributed fungus that causes a fungal infection called Pythiosis is considered an emerging disease in humans and animals. A definitive diagnosis involves clinical, epidemiological, histopathological and molecular correlations. There are no reports of Pythiosis diagnosis involving all these correlations in Colombia. In this study were confirmed Phytiosis in two horses in rural area of Puerto Boyacá (Boyacá, Colombia) presenting single,
\end{abstract}


itching, ulcerated, granulomatous lesions on their limbs with serosanguineous suppuration. Biopsies were analyzed pathologically and histopathologically. Polymerase Chain Reaction (PCR) was carried out from DNA extracted of paraffin blocks. This study report the first definitive diagnosis of phytiosis in Colombia based on the correlation between bio-geography area, the pathological and histopathological findings which included patterns like Splendore-Hoeplii and hyphae with rare septae (2.5 to 6.2 microns diameters of branching angled) along with sequenced DNA that showed $100 \%$ homology with the Pythium insidiosum sequence at the GenBank.

Key words: Pythium insidiosum, Pythiosis, diagnosis.

\section{Introducción}

Pythium sp conforma un genero de hongos oomicetos de los cuales solo la especie Pythium insidiosum causa una enfermedad llamada Pitiosis en personas y animales $(1,2,4,13-21)$. Es considerada una micosis emergente (14). La adquisición de Pitiosis se hace vía zoosporas flageladas motiles en ambientes acuáticos y cenagosos empantanados, en regiones de clima tropical y subtropical $(2,14,22)$.

Las zoosporas de $P$. insidiosum ingresan por lesiones de piel o ingestión (22). En piel intacta, se demuestra, entran por el folículo piloso en equinos y bovinos $(15,23)$; en contacto con tejidos las zoosporas pierden sus flagelos e inician la formación "hifas" en dirección tisular penetrando mecánicamente con la ayuda de enzimas proteolíticas $(4,24,25)$.

En personas, antígenos de $P$. insidiosum de $74 \mathrm{kd}$ dominan la respuesta inmune y en equinos antígenos de $28 \mathrm{Kd}, 30 \mathrm{Kd}$ y $32 \mathrm{Kd}$. Diferencias a nivel de subgrupo de $P$. insidiosum encontrados por análisis filogenéticos explican las diferencias de inmunogenicidad en humanos y equinos (26-28).

Usualmente pitiosis en personas se manifiesta clínicamente restringida a extremidades, como lesiones nodulares cutáneas o subcutáneas, ulceradas con secreción serosanguinolenta $(3,5)$ y en animales se describen restringidas a extremidades y zona ventral toracoabdominal como en ovinos
(18), bovinos(21,23) y equinos (29-32). Adicionalmente con presentación gastrointestinal en caninos $(20,33)$ y respiratoria en felinos $(16,19)$.

En equinos las lesiones frecuentemente presentan masas arenosas amarillentas descritas como formas coralinas llamadas "Kunkers" (16,29-34). Los hallazgos histopatológicos de Pitiosis con histoquímica $\mathrm{H} \& \mathrm{E}$ muestran reacción granulomatosa eosinofila llamada fenómeno de Splendore - Hoepplii en personas (13), ovinos (18), bovinos (23), equinos $(30,31,34)$, felinos (19) y caninos (33).

Los "Kunkers" de la especie equina con H\&E son mayoritariamente agregados de eosinófilos necróticos con desbridados tisulares $(14,30,32,34)$. El diagnóstico de Pitiosis involucra aislamiento del hongo con cultivo a $37^{\circ} \mathrm{C}$, con agar dextrosa - Sabouraud, donde genera colonias blanquecino-amarillentas planas de distribución radial, sin hifas aéreas (3), en el aislamiento la inducción de formación de zoosporas es necesaria (35); o detección sérica de anticuerpos anti $P$. insidiosum por inmunodifusión (36) ELISA, y Western blot $(3,31,11)$; y en tejidos por inmunohistoquímica para detección de de hifas $(18,31)$ e histoquímica para morfometria de hifas con coloración $\mathrm{H} \& \mathrm{E}$ $(13,16,19,20,30,31,33,34)$, PAS $(10,37,38)$ y Gomori (13-14, 16-20, 22-23, 29-34).

El diagnóstico molecular utiliza reacción en cadena de la polimerasa -PCR-, analizando 510 bases 
de los genes $18 \mathrm{~s}$ rDNA de $P$. insidiosum a partir de muestras clínicas o las hifas del material de los cultivos (38-39).

Pitiosis en Sur America es reportada con diagnostico definitivo en animales de Venezuela $(40,41)$ y Brasil $(23,29,43,48)$ y en personas; se registro el primer caso con diagnostico definitivo en Brasil para el ańo 2006 (5). Este trabajo hace un diagnostico definitivo de Pitiosis en Colombia con correlación clínico epidemiológica, patológica, histopatológica y molecular.

\section{Materiales y métodos}

El día 12 de mayo de 2008 dos equinos fueron examinados en consulta particular por el Dr. Gustavo Sanchez, Médico Veterinario en zona rural del Municipio de Puerto Boyacá Departamento de Boyacá - Colombia - Suramérica, coordenadas geográficas 5. 58. latitud norte y 74. 36. longitud oeste Greenwich, a la orilla del Río Magdalena, a 150 metros sobre el nivel del mar; para el año de 2008; según registros públicos ambientales para la fecha del hallazgo con temperatura media entre $25.7^{\circ} \mathrm{C}$ y $29.7^{\circ} \mathrm{C}$, mínima entre 16.8 y $23^{\circ} \mathrm{C}$ y máxima entre 31.4 y $40^{\circ} \mathrm{C}$ (42).

Presentación clínica. Caso uno; equino de raza criolla, genero hembra de 4.5 años de edad, con baja condición corporal, originario de Puerto Boyacá; sus lesiones de 3,5 meses de evolución. Se hallaron en ubicación única en relación con el tegumento de la falange media de miembro posterior izquierdo, Figura 1a.

Caso dos: equino de raza criolla, genero macho de 5 años de edad, con baja condición corporal, originario de Puerto Boyacá. Sus lesiones de 3 meses de evolución también de ubicación única pero en relación con el tegumento de la cara posterior de la articulación falángico-proximal de miembro anterior izquierdo, Figura $1 \mathrm{~b}$.

Las lesiones descritas en ambos casos tuvieron aspecto abultado, homogéneamente ulcerado, con focos en distribución difusa de aspecto "granulomatoso", con secreción serosanguinolenta entre seca y húmeda; a la palpación las masas de aspecto granulomatoso se hallaron depresibles alternadas de masas duras no depresibles, refiriéndose dolor y advirtiéndose prurito intenso sobre los bodes de la lesión llevando a frotar intensamente los artejos contra el macizo facial, no se observó linfadenopatías en los dos casos.

Histopatología. Sin anestesia, por biopsia incisional se tomaron muestras de tejido de aprox. 2 CC. En relación con las masas granulomatosas no depresibles se fijaron en formol al 10\% y se procesaron histotecnicamente con técnicas convencionales en parafina, se hicieron cortes a 4 micras y se colorearon H\&E, (Hematoxilina y Eosina), Acido Peryodico de Schiff (PAS) y Gomori (Plata Metamina de Gomori) como procedió Reis y Noguira (2002) (43).

El análisis histomorfométrico fue realizado por grupo de investigaciones de Procesamiento de Imágenes - PROSEIM - Facultad de MedicinaUniversidad de la Sabana. Utilizando microscopio fotónico marca Leica, con lente de cuadricula milimetrada, calibrado según WHO (44), se midieron los diámetros mayores y menores de las hifas visualizadas en coloración de Gomori, en tres placas cada una con análisis de 20 campos a $100 x$.

El Análisis Molecular se hizo en el Medical Technology Program, Department of Microbiology and Molecular Genetics, Michigan State University tras envío de los bloques de parafina obtenidos del procesamiento histotécnico; allí se extrajo el DNA total de los bloques en parafina que demostraron hifas; el método de extracción como reporta Fredericks and Relman 1999 (45); las secuencias de rDNA (subunidad ribosomal 18S) amplificadas con primers NS1 y NS8 fueron comparadas con las secuencias depositadas en GenBank bajo Pythium insidiosum como procede $(40,46)$. 


\section{Resultados}
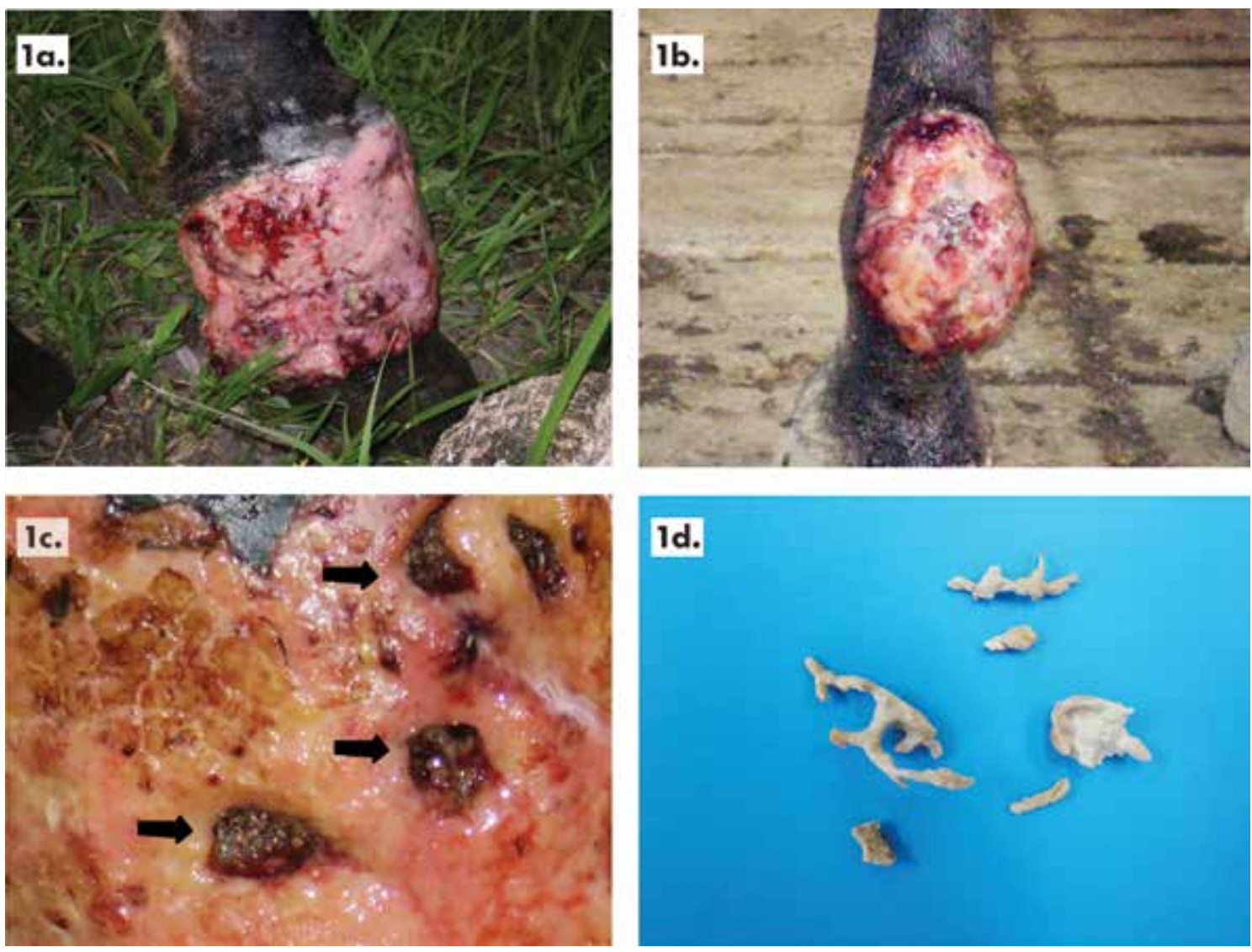

Figura 1. Presentación clínica de las lesiones equinas. Caso uno en miembro anterior (1a). Caso dos en miembro posterior (1b). Acercamiento de las lesiones con aspecto granulomatoso húmedo, ulcerado con secreción serosanguinolenta seca y en flujo, sobre las cuales se hallan masas incrustadas duras de color café (1c). Al corte la lesión ofrece resistencia y al interior se encuentran espacios serosanguinolentos, en cuyo interior se hallan masas sólidas, duras a la manipulación, que desprenden con relativa facilidad, dejando los espacios sin colapsar y con contenido serosanguiolento. Las masas extraídas luego de lavadas revelaron color blanquecino a amarillento, con formas desde cilindróides ramificadas a amorfas, de tamaños variables, oscilando entre 5 a $15 \mathrm{~mm}$ (1d).
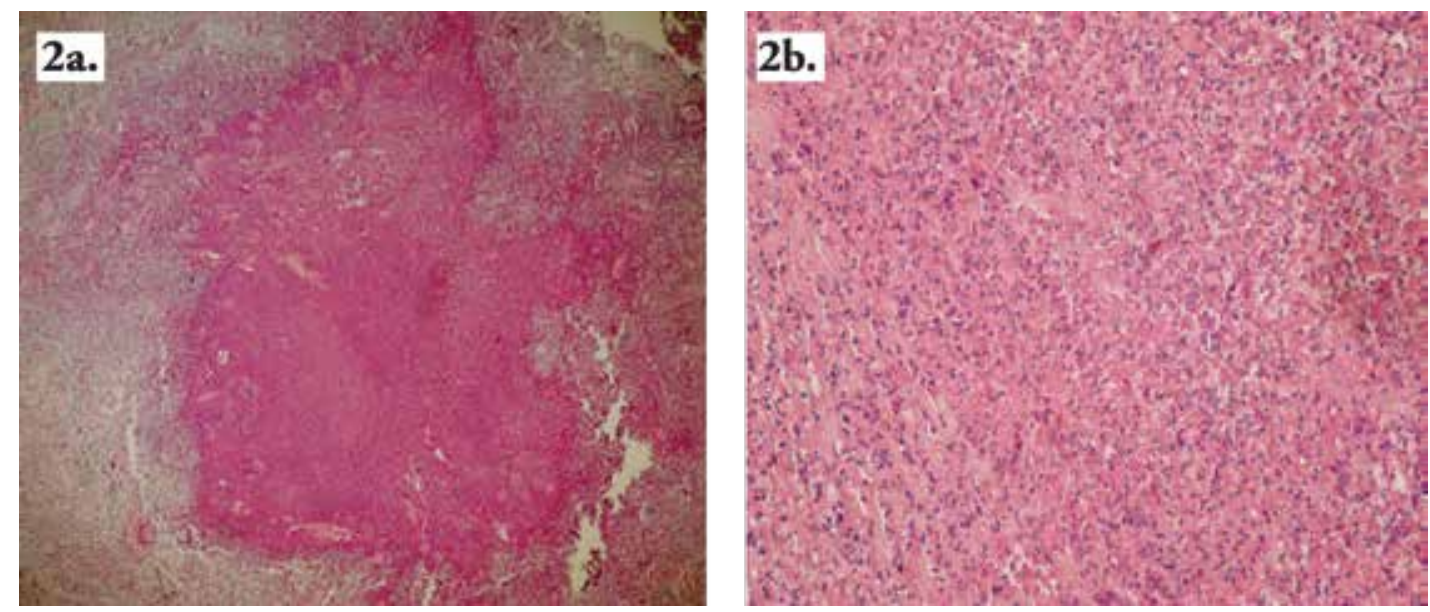

Figura 2. (a) Granuloma con histopatología de Esplendore Hoepplii en coloración H \& E a 5 X (b). Infiltrado de predominio eosinófilo con degeneración colágena en coloración $\mathrm{H} \& \mathrm{E}$ a $40 \mathrm{X}$. 

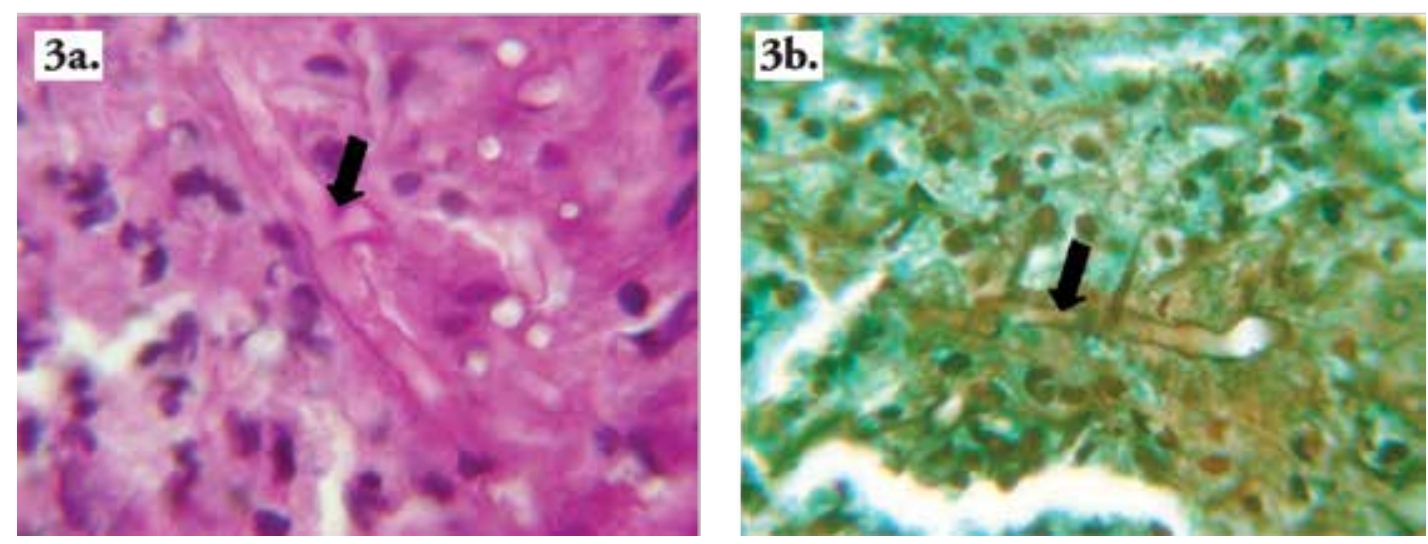

Figura 3. (a) Hifa con ramificación en ángulo recto en coloración de PAS a 100X, (b). hifa con región delgada alternada de región gruesa en coloración de Gomori a 100X.

El DNA extraído y secuenciado por el Dr. Leonel Mendoza PhD y Raquel Vilela PhD en el Biomedical Laboratory Diagnostics Program - Michigan State University mostró que las secuencias amplificadas son compatibles 100\% con las depositadas para la especie Pythium insidiosum en el GenBank.

\section{Discusión}

En el diagnóstico presuntivo e inicial de Pitiosis se correlacionó las coordenadas biogeográficas de la zona del hallazgo, Puerto Boyacá Boyacá - Colombia, reconocidas como zona de vida tropical lluvioso, con temperaturas máximas entre 31.4 y $40^{\circ} \mathrm{C}$ para el año del hallazgo $(2008)(42,47)$ y los animales de los casos clínicos, quienes además de ser originarios de la zona, demostraron estancia permanente en áreas inundables sobre el margen del Río Magdalena en ese último año.

Condiciones ecoepidemiológicas demostradas similares favorecen el ciclo de vida de Pythium sp $(14,29)$, y predominantemente en equinos permiten el desarrollo en extremidades de rápidas lesiones ulceradas, granulomatosas con secreción serosanguinolenta muy pruriginosas, como se describe en esta especie y en ambientes ecoepidemiologicos similares $(17,29,31,52)$.

En nuestros casos el tiempo de evolución de las lesiones reportadas se estimó por anamnesis en 3 y 3,5 meses para cada caso. Aspecto que puede justificar clínicamente la baja condición corporal asociada en esta enfermedad $(30,34)$ y que además coincide con el tiempo, noventa días, para el desarrollo de Pitiosis con generación de baja condición corporal reportada en cuatro equinos del trópico brasilero (34).

Desde el punto de vista anatomopatológico el diagnostico presuntivo de Pitiosis en la especie equina se correlaciona con la presencia de masas muy endurecidas dentro de las lesiones tegumentarias, como incrustadas en ellas, Figura 1c, que al extraerse representa hallazgos similares a masas de aspecto "coralino" llamados "kunkers" descritas en Pitiosis equinas (16,29-34,48-49,51-52), Figura 1d.

Los tamaños de los "kunkers" hallados, entre los rangos de 5 a $15 \mathrm{~mm}$, se encuentran dentro de los tamaños reportados para esta especie (17); y aunque las linfadenopatías en Pitiosis equina son comunes $(14,30$,) en nuestros casos no se evidenciaron distribución asociada al hueso de la falange, la cual es reportada como manifestación anatomopatológica de Pitiosis crónica $(16,30,31)$.

Con respecto al análisis histopatológico y el diagnostico presuntivo de Pitiosis se correlacionó los cortes en coloración de H\&E; Figura 2a y 2b, y la organización granulomatosa compuesta por macrófagos de aspecto epiteloide, células gigantes 
multinucleadas, linfocitos y plasmocitos $(31,34)$, conteniendo en el centro eosinófilos degranulados y células mononucleares rodeando a un material de aspecto eosinofílico, similar al patrón histopatológico granulomatoso de Splendore-Hoeppli, reportado en equinos ante la presencia de Pitiosis $(30,31,34)$. En esta coloración las masas endurecidas coralinas llamadas "kunkers" demostraron ser masivos agregados tisulares necróticos con abundantes eosinófilos degranulados similar a lo referenciado en la literatura para histopatología de equinos con Pitiosis $(30,32,34,52)$.

Con respecto a formas micóticas, no fueron vistas hifas claramente en coloración con $\mathrm{H} \& \mathrm{E}$, como se reporta la literatura $(15,19,20,23,31)$; pero si notables con histoquímica PAS, Figura 3a, como se reportan en otras especies $(37,38)$ y en personas(10) y con histoquímica de Gomori como se diagnostica en equinos (29-32,43,52), Figura 3 b.

Con coloración de Gomori, las hifas halladas fueron mas abundantes en la periferia de los masas coralinas endurecidas llamadas "kunkers" $(30,32,34,43,52)$. Las hifas halladas morfometricamente fueron similares a las descritas en equinos y sugestivas de $P$. insidiosum, esto es; con ramificaciones en ángulo recto, Figura 3a, $(29,30,34,43,49,52)$, raramente septadas $(29,31,34,43,52)$ y con diámetro hifa oscilando entre 2,5 a 6,2 micras, Figura 3b, ó de 2 a 6 micras $(14,29,31)$.

La variabilidad de los rangos entre hifas puede relacionarse por distribución no paralela de las paredes de las mismas (48). Incluso se reportan diámetros de 3,5- 5.5 micras $(30,33)$.

El diagnóstico diferencial de Pitiosis en la especie equina es orientada sobre micosis causadas por Basidiobolus ranarum y Conidiobolus coronatus, cuyos diámetros de hifas para el primero es $5.1 \mathrm{a}$ 20.5 micras y 5.1 a 12.8 micras para el segundo $(50,34)$, rangos que están por encima de los hallados en los dos casos.
Adicionalmente se descartaron estas micosis histopatologicamente porque $B$. ranarum relaciona mas hallazgos edematosos que Pitiosis, la cual es altamente fibrotico y celular. Por otro lado, anatomopatológicamente $C$. coronatus restringe su presentación sobre región nasal (30) y $P$. insidiosum predomina en extremidades y zona toracoabdominal ventral en esta especie (29-32).

Otro diagnostico diferencial infeccioso fuera de micosis en equinos se establece con parasitosis como habronemiasis que se describe también desarrolla "kunkers", y miasis, que desarrolla reacciones granulomatosa intensas $(30,52)$, pero se descartaron al no hallarse formas helmínticas o larvas respectivamente tanto clínica como anatomopatológicamente e histopatologicamente.

Finalmente, en esta especie, el sarcoide equino considera la literatura hace parte de los diagnósticos diferenciales de Pitiosis dada la progresión de las lesiones de Pitiosis simulando un cuadro tumoral por lo cual Pitiosis es también llamada cáncer de los pantanos $(31,29,52)$. Sin embargo, se descartó por la no visualización histopatológica de formas celulares neoplásicas como "pseudoepiteliomas” típicos del sarcoide equino (51).

El diagnóstico definitivo de Pitiosis con el DNA extraído se confirmó, cuando las secuencias amplificadas manifestaron $100 \%$ de compatibilidad con las depositadas para la especie Pythium insidiosum en el GenBank. La confirmación de Pitiosis en Colombia sugiere que en nuestro medio zonas de vida definidas como tropicales lluviosas, puede favorecer la presencia de P.insidiosum con capacidad infectante.

\section{Agradecimientos}

Los autores expresan su agradecimiento al Dr. Gustavo Sanchez, Médico Veterinario. Puerto Boyacá -Departamento de Boyacá - Colombia. Dr. Leonel Mendoza PhD, Director e Investigador del Biomedical Laboratory Diagnostics Program - Michigan State University. Dra. Raquel Vilela 
PhD. Investigadora en el Biomedical Laboratory Diagnostics Program - Michigan State University

\section{Referencias}

1. De Cock AW, Mendoza L, Padhye AA, Ajello L, Kaufman L. Pythium insidiosum sp. nov., the etiologic agent of pythiosis. J Clin Microbiol. 1987;25: 344-9.

2. Mendoza L, Ajello L, McGinnis MR. Infection caused by the Oomycetous pathogen Pythium insidiosum. J Mycol Med. 1996;6:151-64.

3. Krajaejun T, Sathapatayavongs, B, Chaiprasert, A. Srimuang, S. Do You Know Human Pythiosis? Infect Dis Antimicrob Agents. 2008;25(1):45-51.

4. Mendoza L, Hernandez F, Ajello L: Life cycle of the human and animal oomycete pathogen Pythium insidiosum. J Clin Microbiol. 1993;31(11):2967-2973.

5. Marques SA, Bagagli E, Sandra MG, Bosco Rosangela M P, Camargo Mariangela EA, et al. Pythium insidiosum: report of the first case of human infection in Brazil. An Bras Dermatol. 2006;81(5):483-5.

6. Thianprasit M, Chaiprasert A, Imwidthaya P.Human pythiosis. Curr. Top. Med. Mycol.1996;7:43-54.

7. Krajaejun T, Sathapatayavongs B, Pracharktam R. Clinical and epidemiological analyses of human pythiosis in Thailand. Clin Infect Dis 2006;43:569-76.

8. Prasertwitayakij N, Louthrenoo W, Kasitanon N, Thamprasert K. Human pythiosis, a rare cause of arteritis: case report and literature review. Semin Arthritis Rheum. 2003;33:204-14.

9. Heath JA, Kiehn TE, Brown AE, LaQuaglia MP, Steinherz LJ, Bearman G, et al. Pythium insidiosum pleuropericarditis complicating pneumonia in a child with jeukemia. Brief Report. CID. 2002;35:60-4.

10. Pfaller MA, Diekema D J. Unusual Fungal and Pseudofungal Infections of Humans. J. Clin. Microbiol. 2005;43:1495-1504.

11. Vanittanakom N, Supabandhu J,Khamwan C, Praparattanapan J, Thirach S, Prasertwitayakij N, et al. Identification of emerging human-pathogenic Pythium insidiosum by serological and molecular assay-based methods. J. Clin. Microbiol. 2004; 42(9): 3970-3974.

12. Ribes JA, Vanover-Sams CL, Baker DJ. Zygomycetes in human disease Clin Microbiol Reviews. 2000;13(2):236-301.

13. Bosco SM, Bagagli E, Araújo JP, Candeias JM, de Franco MF, Alencar ME, et al. Human pythiosis Brazil. Emerg Infect Dis. 2005;11:715-8.

14. Santurio JM, Alves SH, Pereira DB, Argenta JS, Pitiose: uma micose emergente. Acta Scientiae Veterinariae. 2006;34(1):1-14.

15. Miller RI. Investigations into the biology of three 'phycomycotic' agents pathogenic for horses in Australia. Mycopathologia. 1983;81:23-28.

16. Stephens B A, Tarpley HL, Rakich PM, Latimer KS, Johnson ME, Bain PJ. Oomycosis: Pythiosis in the dog, horse and cat and Lagenidiosis in the dog. Accesado Julio 12 de 2008.Disponible de http://www.vet.uga.edu/VPP/clerk/stephens/index.php

17. Biava J B, Ollhoff DB, Gonçalves RC, Biondo AW. Zygomycosis in equines. A review. Rev Acad Curitiba. 2007;(5)3: 225-230.
18. Tabosa IM, Riet-Correa F, Nobre VMT, Azevedo EO J, ReisJunior L, Medeiro RMT. Outbreaks of pythiosis in two flocks of sheep in northeastern Brazil. Vet Pathol. 2004;(41):412-415

19. Alvin C, Camus A, Grooters M, Roberto F. Aquilar granulomatous pneumonia caused by Pythium insidiosum in a central American jaguar, Panthera onca. J Vet Diagn Invest. 2004;(16):567-571.

20. Mendoza L, Arias M, Colmenarez V, Perazzo Y, Intestinal canine pythiosis in Venezuela confirmed by serological and sequencing análisis Mycopathologia. 2005;(159): 219-222.

21. Miller RI, Olcott BM, Archer M. Cutaneous pythiosis in beef calves. J Am Vet Med Assoc. 1985 (186): 984-986.

22. Schurko AM, Mendoza L, Arthur WA,. de Cock M, Bedard J, Klassen M. Development of a species-specific probe for Pythium insidiosum and the diagnosis of pythiosis. J Clin Microbiol. 2004;42(6):2411-2418.

23. Santurio JM, Monteiro AB, Leal AT, Kommers GD de Sousa DS, Catto JB. Cutaneous pythiosis insidiosi in calves from the pantanal region of Brazil. Mycopathologia. 1998;(141):123-125.

24. MacDonald E, Laurie Millward JP, Ravishankar NP. Biomechanical interaction between hyphae of two Pythium species (Oomycota) and host tissues. Fung Genet Biol. 2002;37:245-249.

25. Ravishankar JP, Davis CM, Davis DJ, MacDonald E, Makselan SD, Millward L, et al. Mechanics of solid tissue invasion by the mammalian pathogen Pythium insidiosum. Fung Genet Biol. 2001;34:167-175.

26. Krajaejun T, Kunakorn M, Pracharktam R, Chongtrakool P, Sathapatayavongs B, Chaiprasert A . et al. Identification of a novel 74-kilodalton immunodominant antigen of Pythium insidiosum recognized by sera from human patients with pythiosis. J Clin Microbiol. 2006;44(5):1674-1680.

27. Mendoza L, Nicholson V, Prescott JF. Immunoblot analysis of the humoral immune response to Pythium insidiosum in horses with pythiosis. J. Clin. Microbiol. 1992;30:2980-2983.

28. Schurko A M, Mendoza L, de Cock A. W, Klassen G. R. Evidence for geographic clusters: Molecular genetic differences among strains of Pythium insidiosum from Asia, Australia and the Americas are explored. Mycologia. 2003; 95(2): 200-208.

29. Santurio, JM, Catto JB, Comastr JA, Leal AT, Leal ABM. Ferida da moda: epidemiologia, diagnóstico, tratamento e experiencia com eqüinos infectados no pantanal. Comunicado Tecnico 34. 2004. Ministério da Agricultura, Pecuária e Abastecimento. 1.ed., Brazil . Accesado Julio 20 de 2008.Disponible de: http://www.cpap.embrapa.br/publicacoes/online/ COT34.pdf

30. Headley SA, Arruda Jr HN, Bett LV, Cutaneous pythiosis in a slaughtered horse: a case report. Arq. Inst. Biol. 2002;69(4):109-112.

31. Poole HMM, Brashie MK, Equine cutaneous pythiosis. Compendium. 2003; 25(3):229-235.

32. Luvizari FH, Lehmkuhl RC, Santos IW, Pitiose equina no estado do paraná - primeiro relato de caso Arch Vet Scien 2002;7(2):99-102.

33. FischerJF,Pace LW, Turk JR, Kreeger JM, Miller MA,Gosser HS. Gastrointestinal pythiosis in Missouri dogs: eleven cases J Vet Diagn Invest. 1994; 6:380-382.

34. Headley HA. Arruda Jr HN, Equine cutaneous pythiosis: a report of four cases. Ciência Rural. 2004;34(1):289-292. 
35. Pereira DSB, Santurio JM, AlvesSH, Argenta JS, Cavalheiro AS, et al Zoosporogênese in vitro entre isolados do oomiceto Pythium insidiosum. Ciência Rural. 2008;38 (1):143-147.

36. Kaufman L, MendozaL, Standard PG. Immunodiffusion test for serodiagnosing subcutaneous zygomycosis. J Clin Micribiol. 1990;28:1887-1890.

37. Rivierre C, Laprie C, Guiard-Marigny O, Bergeaud P, Berthelemy. M. Guillot M. Pythiosis in Africa. J. Emering Infect Dis. 2005;11(3):479-481.

38. Kaufman L. Penicilliosis marneffei and pythiosis: Emerging tropical diseases. Mycopathologia. 1998;143:3-7.

39. Badenoch PR, Coster DJ, Wetherall BL, Brettig HT, Rozenbilds AM, Drenth A, et al. Pythium insidiosum keratitis confirmed by DNA sequence analysis. Br J Ophthalmol. 2001;85:496.

40. 40. Mendoza L. Arias M, Colmenarez V, Perazzo Y Intestinal canine pythiosis in Venezuela confirmed by serological and sequencing análisis. Mycopathologia. 2005;159: 219-222

41. 41. Luis-León JJ, Pérez RC, Vivas JL, Mendoza L, Alonso FT. Confirmación de Pythium insidiosum como agente etiológico de la Granulomatosis Enzoótica Bovina mediante análisis de secuencia. Salus online. 2009;12 (Sup. 1):205-215.

42. Plan de Desarrollo. Pueblo y Democracia 2008-2011 Municipio de Puerto Boyacá. Boyaca-Colombia. Acuerdo No 013 del 31 de Mayo de 2008. Accesado Mayo 8 de 2010Disponible en: http://puertoboyaca-boyaca.gov.co/apc-aa-files/36353061306433633731633432313233/PLAN DE DESARROLLO 20082011 PUERTO BOYACA.pdf.

43. Reis Júnior JL, Nogueira RHG, Estudo anatomopatológico e imunoistoquímico da pitiose em eqüinos naturalmente infectados. Arq Bras Meda Vet Zoot. 2002;54(4):358-365.
44. World Health Organization (WHO). Calibrating the microscope. In: Basic laboratory methods in medical parasitology, 1991. Accesado Enero 22 de 2011Disponible de: http:// whqlibdoc.who.int/publications/9241544104_(part1).pdf.

45. Fredricks D N, Relman D A. Application of polymerase chain reaction to the diagnosis of infectious diseases. CID. 1999;29: 475-488.

46. Reis JL, Quiroz-deCarvalho CC, Girao-Nogueira RH, Lemos LS, Mendoza L. Disseminated pythiosis in three horses. Vet Microbiol. 2003;96:289-295.

47. Holdridge LR. Grenke WC. Hatheway WH. Liang T. Tosi JA. Forest environments in tropical life zones, a pilot study. Pergamon Press, Oxford. 1971. p 78-81

48. 5 year-old, female quarter horse. Enhanced wednesday slide Conference Online. Department of Defense Veterinary Pathology Residency. AFIP. March 21, 2007. Conf 18 - Case 3. Accesado Julio 12 de 2008. Disponible de http://vp4.afip.org/ wsco/wsc showcase2.php?id=16.

49. Grooters AM, Whittington A, Lopez MK, Michelle N. Boroughs, AFR Evaluation of microbial culture techniques for the isolation of Pythium insidiosum from equine tissues.J Vet Diagn Invest. 2002;14:288-294.

50. Miller RI, Campbell RSF. The comparative pathology of equine cutaneous phycomycosis. Vet. Pathol. 1984;21: 325-332.

51. Monteiro Leal AB, Alexandre T, Leal, JM, Santurio GD, Kommerse J, Catto B. Pitiose eqüina no Pantanal brasileiro: aspectos clínicopatológicos de casos típicos e atípicos. Pesq. Vet. Bras. 2001;21(4):151-156.

52. Sallis V, Pereira DB, Raffi MB. Pitiose cutânea em eqüinos: 14 casos Ciência Rural. 2003; 33(5)1-8. 